\title{
RESILIENCIA E INTELIGENCIA EMOCIONAL. VARIABLES DE PROTECCIÓN EN MENORES RESIDENTES EN RECURSOS DE PROTECCIÓN
}

\author{
RESILIENCE AND EMOTIONAL INTELLIGENCE: VARIABLES IN PROTECTION \\ OF MINORS WHO RESIDE IN JUVENILE FACILITIES
}

\section{RESILIÊNCIA E INTELIGÊNCIA EMOCIONAL. VARIÁVEIS DE PROTEÇÃO EM MENORES RESIDENTES EM RECURSOS DE PROTEÇÃO}

\author{
Raúl CARRETERO BERMEJO
}

Universidad Complutense de Madrid (UCM)

PALABRAS CLAVE:

resiliencia

inteligencia

emocional

menores

educación social

intervención social

centros de

protección
Fecha de recepción: 12.1.2018

Fecha de revisión: 6.111.2018

Fecha de aceptación final: 26.III.2018
RESUMEN: Los jóvenes residentes en centros de protección se encuentran con la situación de que tienen que abandonar el recurso al cumplir los 18 años y pasar a vivir de una forma completamente independiente. En este complicado contexto nos preguntamos acerca de las herramientas con las que cuentan para enfrentarse a esta nueva situación, presentándose la Inteligencia Emocional y la Resiliencia como dos variables relacionadas con el éxito (escolar, social, laboral y relacional) y la superación de situaciones adversas.

Así, los objetivos de nuestro estudio eran conocer las puntuaciones en Inteligencia Emocional y Resiliencia de estos menores residentes en centros de protección y compararlas con las puntuaciones de menores que no residen en este tipo de recursos. Esperábamos encontrar diferencias significativas entre ambos grupos, donde los menores que residen en algún recurso de protección obtienen puntuaciones significativamente inferiores en estas dos variables a los menores que residen en su unidad familiar y sin vinculación y/o relación alguna con los servicios sociales.

Para el diseño se ha decidido seleccionar a jóvenes de ambos grupos que están estudiando $3^{\circ}, 4^{\circ}$ de ESO y $1^{\circ}, 2^{\circ}$ de Bachillerato para controlar variables relacionadas con nivel académico, formación y motivaciones familiares. Se ha construido un cuestionario que recoge información sociofamiliar e incluye el TMMS-24 (Fernández-Berrocal, Extremera \& Ramos, 2004) para recoger la información relativa a las creencias de estos jóvenes sobre su inteligencia emocional y el RESI-m (Palomar \& Gómez, 2010) para recoger la información relativa a Resiliencia. 


\begin{tabular}{|c|c|}
\hline & $\begin{array}{l}\text { Los resultados muestran que existen diferencias significativas en las puntuaciones ob- } \\
\text { tenidas por ambos grupos. Los menores residentes en centros de protección obtienen pun- } \\
\text { tuaciones significativamente inferiores en Inteligencia Emocional y en Resiliencia, lo que } \\
\text { supone contar con menos herramientas para hacer frente a una situación, en principio, } \\
\text { más adversa. }\end{array}$ \\
\hline $\begin{array}{l}\text { KEY WORDS: } \\
\text { resilience } \\
\text { emotional } \\
\quad \text { intelligence } \\
\text { minors } \\
\text { social education } \\
\text { social intervention } \\
\text { juvenile facilities }\end{array}$ & $\begin{array}{l}\text { ABSTRACT: Youths who reside in juvenile facilities find themselves with having to leave the } \\
\text { centre when they reach } 18 \text { years old to become completely independent. In this difficult back- } \\
\text { ground, we ask ourselves what tools they are equipped with when facing this new situation. } \\
\text { We find Emotional Intelligence and Resilience as two variables associated with their success, } \\
\text { i.e. academic, social, professional at a relationship level, and in overcoming adverse situations. } \\
\text { Thus, the aim of our study was to find out their scores in Emotional Intelligence and } \\
\text { Resilience and compare them to scores of minors who live outside of these centres. The ex- } \\
\text { pectation was to find a significant difference between the two groups, where the minors who } \\
\text { reside in a juvenile facility would get significantly lower scores in the two variables than the } \\
\text { minors who live within family units and have no relation to social services. } \\
\text { In the design of the study, youths in their } 3 \text { rd and } 4 \text { th year of the secondary school and } \\
\text { the higher secondary ist and } 2 \text { nd courses were selected from both groups to examine varia- } \\
\text { bles in relation to their academic level, background and family motivations. A questionnaire } \\
\text { was designed to gather social and family information data; it included the TMMS-24 test } \\
\text { (Fernández-Berrocal, Extremera \& Ramos, 20o4) to probe their impressions on their emo- } \\
\text { tional intelligence and the RESI-m test (Palomar \& Gómez, 2O10) to find information related } \\
\text { to Resilience. } \\
\text { The results show significant gaps in the scores obtained by the two groups. The minors } \\
\text { who live in juvenile facilities qualify for significantly lower scores in Emotional Intelligence and } \\
\text { Resilience, which implies they have fewer resources available to confront an adverse situa- } \\
\text { tion, as abandoning the centre when they become of age could be. }\end{array}$ \\
\hline $\begin{array}{l}\text { PALAVRAS-CHAVE: } \\
\text { intervenção } \\
\text { psicoeducativa } \\
\text { adolescência } \\
\text { comportamento } \\
\text { antissocial } \\
\text { programas baseados } \\
\text { na evidência } \\
\text { profissionais }\end{array}$ & $\begin{array}{l}\text { RESUMO: Os jovens que residem em instalações juvenis se vêem obrigados a deixar o centro } \\
\text { quando chegam aos } 18 \text { anos para se tornarem completamente independentes. Neste contex- } \\
\text { to difícil, nos perguntamos com que ferramentas eles estão equipados para enfrentar essa } \\
\text { nova situação. Encontramos Inteligência Emocional e Resiliência como duas variáveis associa- } \\
\text { das ao seu sucesso, ou seja, acadêmico, social, profissional em nível de relacionamento e na } \\
\text { superação de situações adversas. } \\
\text { Assim, o objetivo do nosso estudo foi descobrir suas pontuações em Inteligência Emo- } \\
\text { cional e Resiliência e compará-las a dezenas de menores que moram fora desses centros. A } \\
\text { expectativa era encontrar uma diferença significativa entre os dois grupos, onde os menores } \\
\text { que residem em uma instalação juvenil receberiam pontuações significativamente mais baixas } \\
\text { nas duas variáveis do que os menores que vivem dentro das unidades familiares e não têm } \\
\text { relação com os serviços sociais. } \\
\text { No delineamento do estudo, jovens de 3o e } 40 \text { ano do ensino médio e de } 20 \text { e } 20 \text { ano do } \\
\text { ensino médio foram selecionados de ambos os grupos para examinar variáveis em relação ao } \\
\text { seu nível acadêmico, antecedentes e motivações familiares. Um questionário foi elaborado } \\
\text { para coletar dados de informações sociais e familiares; incluiu o teste TMMS-24 (Fernández- } \\
\text {-Berrocal, Extremera \& Ramos, 20o4) para investigar suas impressões sobre sua inteligência } \\
\text { emocional e o teste RESI-m (Palomar \& Gómez, 2010) para encontrar informações relaciona- } \\
\text { das à Resiliência. } \\
\text { Os resultados mostram lacunas significativas nos escores obtidos pelos dois grupos. Os } \\
\text { menores que moram em instalações juvenis se qualificam para pontuações significativamente } \\
\text { mais baixas em Inteligência Emocional e Resiliência, o que implica que eles têm menos re- } \\
\text { cursos disponíveis para enfrentar uma situação adversa, pois o abandono do centro quando } \\
\text { atingem a idade poderia ser. }\end{array}$ \\
\hline
\end{tabular}

\section{Introducción}

Los menores tutelados por la Administración y que residen en alguno de los centros de protección responsabilidad de esta administración se encuentran con la situación, normalmente complicada, de que con la mayoría de edad tienen que abandonar el recurso en el que vivían y tenían todas sus necesidades básicas cubiertas para vivir de manera completamente independiente. Existen recursos especializados para facilitar el tránsito a la vida autónoma aunque, sin embargo, estos recursos no tienen la capacidad de atender a todas las personas que se encuentran con esta situación, por lo que la mayoría de menores que dejan su recursos de protección tienen que enfrentar este cambio sin ayuda institucional y/o familiar. En esta situación, cuanto menos, complicada, la Inteligencia Emocional y la Resiliencia emergen como dos herramientas poderosas para que estos menores puedan superar esta situación con éxito (Muñoz-Silva, 2012; Palma-García \& Hombrados-Mendieta, 2013). 
Diversos estudios presentan a la Inteligencia Emocional como una de las principales variables predictoras del éxito académico (Ferragut \& Fierro, 2012; Jiménez Morales \& López Zafra, 2009), la inserción e integración laboral (Solano-Gómez, 2013) y del bienestar psicológico (Salguero, Palomera y Fernández-Berrocal 2012). De igual forma, la Resiliencia aparece como una variable que correlaciona positivamente con el éxito académico (Gaxiola, Gónzalez, Contreras \& Gaxiola, 2012) recuperación tras vivir una situación de riesgo o especialmente adversa (Rutter, 2007) o como fundamental para el desarrollo social (Masten, 2007). También se muestra como una variable relevante en la recuperación familiar ante situaciones de exclusión (López, Rosales, Chávez, Byrne \& Cruz, 2009; Gómez \& Kotliarenco, 2010). Por otra parte, en la propia definición de Resiliencia como "la capacidad, fruto de la interacción de diferentes variables personales con factores ambientales, que permite al individuo enfrentarse y resolver, de manera adecuada e integrada en su entorno cultural, diferentes situaciones de adversidad, riesgo o traumáticas por diferentes motivos, permitiéndole alcanzar una situación normalizada y adaptada a su medio cultural" (Carretero-Bermejo, 2010) aparece explícitamente su relevancia en la resolución de situaciones adversas (Vera, Carbelo \& Vecina, 2007).

En este contexto especialmente duro, la Inteligencia Emocional y la Resiliencia se presentan como variables de protección especialmente importantes y relevantes para este grupo de menores, en comparación con aquellos que residen con sus familias (considerando las diversas situaciones familiares posibles) que, en principio, disponen de más recursos y ayudas para enfrentar esta, ya de por sí difícil, situación de cambio.

Las habilidades que forman el constructo de Inteligencia Emocional varían, en algunos casos de forma significativa, según el modelo desde el que se desarrolle el estudio. La literatura científica presenta dos grandes modelos de Inteligencia Emocional. Los modelos mixtos y los modelos de habilidad. Si nos acercamos a la Inteligencia Emocional como una teoría de la inteligencia encontramos una clara diferenciación entre los modelos de Inteligencia Emocional mixtos y los puros o de habilidad. Los modelos de habilidad, o modelos puros, se concentran en los aspectos cognitivos que cada persona utiliza en su procesamiento emocional de la información. Los modelos mixtos contienen una diversidad de aptitudes, comportamientos, y rasgos de personalidad.

El presente estudio se ha desarrollado desde la perspectiva teórica del modelo de habilidad de Mayer y Salovey (1997.) Dicho modelo centra la atención en los aspectos cognitivos, quedando la Inteligencia Emocional descrita en este modelo como "la habilidad para percibir, valorar y expresar la emoción adecuadamente y adaptativamente; la habilidad para acceder y/o generar sentimientos que faciliten el pensamiento; la habilidad para entender las emociones y la cognición emocional; y la habilidad para regular las emociones para promover el crecimiento intelectual y emocional" (Mayer \& Salovey, 1997).

Este enfoque presenta a la Inteligencia Emocional desde una perspectiva cercana a los modelos de inteligencia del procesamiento de la información (Mayer \& Salovey, 1997; Salovey \& Grewlar, 2006) quedando el modelo de Inteligencia Emocional conformada por cuatro aptitudes relacionadas entre sí. 1a) Capacidad para percibir y expresar las emociones de forma precisa: Percepción y expresión de las emociones. 2a) Capacidad para encauzar las emociones de modo que faciliten el pensamiento y el razonamiento (uso inteligente de las emociones). 3a) Capacidad para comprender las emociones, especialmente el lenguaje de las emociones (comprensión emocional). Y $4^{\text {a) }}$ capacidad para controlar las propias emociones y las de los demás (manejo de las emociones)

Estas cuatro habilidades que conforman el modelo de Mayer y Salovey están íntimamente relacionadas entre sí, de tal forma que el deficiente desempeño de una de ellas influye directamente en el funcionamiento de las demás. Parece evidente que no podremos regular una emoción sino somos capaces de percibirla y reconocerla. Desde este modelo de habilidad la Inteligencia Emocional se muestra en dos vertientes: Por un lado en lo referido a como utilizar la Inteligencia Emocional con uno mismo, lo que se denomina Inteligencia Intrapersonal, y por otro en lo referido a la utilización de la Inteligencia Emocional con los demás, denominada Inteligencia Interpersonal.

Los estudios sobre Resiliencia persiguen conocer las variables por las que algunas personas son capaces de superar las situaciones adversas a las que tienen que hacer frente, al tiempo que otras personas se muestran incapaces de superar situaciones similares. Existía la creencia de que estas respuestas resilientes eran la excepción, e inclusos patológicas, considerando como normal el hecho de no ser capaz de superar estas situaciones de adversidad.

Actualmente, desde diversos y numerosos estudios científicos, se presenta una realidad bien diferente, donde la Resiliencia aparece como una respuesta muy habitual, donde su aparición no indica patología, sino un ajuste saludable ante las diferentes situaciones de adversidad a las que cada persona se enfrenta a lo largo de su clico vital. La 
Resiliencia es, por tanto, común, frecuente y habitual y surge de funciones y procesos de adaptación normales en los seres humanos (Cyrulnick, 2001)

El constructo de Resiliencia es tratado y definido de manera diferente entre investigadores americanos y europeos. Desde la perspectiva europea, la mayor parte de ellos franceses, la Resiliencia queda relacionada con el concepto de crecimiento postraumático, dado que definen Resiliencia al mismo tiempo como la capacidad de superar indemne una situación de adversidad, aprender de ella y, además, mejorar determinadas habilidades o capacidades como fruto de este proceso.

Desde la perspectiva americana se concreta o restringe el contenido de la definición de Resiliencia para hacer referencia únicamente al proceso de afrontamiento que cada persona implementa para superar la situación de adversidad y mantenerse intacta, diferenciándolo de esta manera del concepto de crecimiento postraumático. La Resiliencia refleja la habilidad o capacidad para mantener un equilibrio estable durante todo el proceso (Bonnano, 2004).

la Resiliencia se presenta como un constructo inferido por un lado de la existencia de una situación adversa y por otro de la definición de crecimiento y normalización de una situación.

Hablar de Resiliencia requiere el cumplimiento de tres características esenciales: en primer lugar habrá que determinar la existencia de una amenaza, de una situación adversa o de riesgo para la persona; la segunda consiste en que la persona adquiera, a partir de sus interacciones psicológicas con su ambiente, la tendencia a la superación y posterior adaptación ante las adversidades; y, por último, es necesario mostrar la competencia en una tarea del desarrollo de acuerdo con su edad y con el contexto sociocultural de la persona (Gaxiola, Frías, Hurtado, Salcido \& Figueroa, 2011).

Aunque la Resiliencia se manifiesta en las personas, es la resultante de la interacción entre variables personales, como el cociente intelectual, las habilidades sociales o la propia inteligencia emocional, y variables ambientales o contextuales como la familia, los grupos de referencia, la pertenencia y participación en asociaciones o la escuela (Carretero-Bermejo, 2010). Por ello, no es posible hablar de variables suficientes, y tampoco necesarias, que determinen o den como resultado la aparición de la Resiliencia. Hablamos, en cambio, de factores de riesgo, como aquellos que aumentan la posibilidad de riesgo o no superación de una situación de adversidad y de factores protectores, para referirnos a aquellos factores que aumentan la posibilidad de éxito y superación de la situación de adversidad (Carretero-Bermejo, 2010; Masten, 2007).

\section{Justificación y objetivos}

Conocer la puntuación en Inteligencia Emocional y Resiliencia de los menores residentes en recursos de protección nos puede proporcionar información muy valiosa sobre las herramientas con las que cuentan estos menores para hacer frente a su realidad vital y puede, también, explicar las dificultades y problemas que aparecen en estos menores, donde, según la percepción de los profesionales de los servicios sociales, muchos de ellos terminan en recursos de reforma, condenados, en prisión y repitiendo modelos de exclusión.

Además, la comparación de sus puntuaciones con las puntuaciones de jóvenes que nada tienen que ver con los servicios sociales nos puede dar información acerca del papel de los recursos de protección en la educación, desarrollo y satisfacción de necesidades de los menores que tienen a su cargo, así como de posibles necesidades de formación profesional para los trabajadores de esos recursos.

Esta información puede estar en la base, y justificar, medidas concretas en cuanto, primero, la formación y experiencia necesaria para las personas que trabajan en estos recursos. Segundo, la organización y funcionamiento de estos recursos. Tercero, las variables y dimensiones a tener en cuenta en los procesos de evaluación del funcionamiento de los recursos.

Los objetivos de nuestro estudio eran: en primer lugar, conocer las puntuaciones en Inteligencia Emocional y Resiliencia de las personas menores de edad que residen en un centro de protección tutelado por su Comunidad Autónoma de referencia. En segundo lugar comparar estas puntuaciones con las puntuaciones de menores de edad que viven con sus familias y no han tenido ninguna medida de protección ni son objeto de intervención en ningún recurso de Servicios Sociales. Por último encontrar información sobre las variables, en nuestro estudio, la Inteligencia Emocional y la Resiliencia, que puedan ayudarnos a comprender y a mejorar los procesos de cambio a los que se enfrentan las personas en esta situación de protección.

Derivadas de estos objetivos, nuestras hipótesis de trabajo eran: 1). Las personas menores de edad que residen en un centro de protección de menores obtienen puntuaciones significativamente más bajas en Inteligencia Emocional que las personas menores de edad que residen con sus familias y no tienen ninguna medida de protección ni son objeto de intervención en Servicios 
Sociales; 2). las personas menores de edad que residen en un centro de protección de menores obtienen puntuaciones significativamente más bajas en Resiliencia que las personas menores de edad que residen con sus familias y no tienen ninguna medida de protección ni son objeto de intervención en Servicios Sociales.

\section{Metodología}

Para el desarrollo de este trabajo se seleccionó una muestra de 486 personas mediante un muestreo no probabilístico por conveniencia, con un $5,66 \%$ de margen de error, controlando el número de participantes por sexo, edad, estudios y ciudad de residencia.

Después de revisar todos los cuestionarios hemos contado finalmente con 408 personas, quienes completaron correctamente la prueba utilizada en este diseño de investigación. Las personas que han participado en este diseño cuentan con edades comprendidas entre los 14 y los 18 años, siendo la edad media de nuestra muestra de 16,89 años. De ellas, 198 eran mujeres $(48,5 \%)$ y 210 hombres (51,5\%).

En nuestra muestra 138 de las personas que han participado $(36,8 \%)$ tienen declarada una medida de protección y viven en un centro de protección de menores tutelado por la administración y las 270 restantes $(63,2 \%)$ vive con sus familias $\sin$ ningún tipo de medida de protección ni trabajo de intervención desde los servicios sociales.

Por cuestiones relativas a la confidencialidad, legislación vigente sobre el derecho a la intimidad y la situación concreta de este colectivo de menores residentes en centros de protección hemos decidido no publicar datos relativos a ciudades, comunidades y/o provincias, centros de protección o institutos públicos que han participado en el desarrollo de este trabajo. Esta medida fue, además, una petición expresa y una condición para la participación en este estudio de los responsables políticos y técnicos de los recursos de protección que finalmente han participado.

En cuanto al procedimiento llevado a cabo en la implementación de este trabajo, se ha pedido, en primer lugar, mantener una entrevista para tener ocasión de explicar y matizar el contenido del diseño y su finalidad a los responsables técnicos y políticos de los centros de protección que han querido participar. Se ha pedido, por escrito, autorización para poder recoger la información sobre las personas que convivían en cada recurso a la administración, como responsable y tutora legal de los menores. Se ha decidido, junto con los técnicos, que en cada centro se designará a una educadora o educador y fuera ella quien administrara el cuestionario para evitar, en la medida de lo posible, distorsionar las rutinas de centro y no interferir en la vida del mismo.

Simultáneamente se contactó con los equipos directivos en los Institutos situados en las localidades de los centros de protección para poder también recoger información en sus centros. En este caso, se optó por que fueran los responsables del diseño quienes recogieran la información en las aulas en horario de tutoría. Previamente el tutor de cada curso que decidió participar entregó un modelo de autorización a sus alumnos para que las familias estuvieran informadas y dieran su consentimiento de participación en el diseño. La participación en todas las fases del diseño ha sido voluntaria y anónima, para garantizar el derecho a la intimidad y el respeto a la legislación vigente también de los menores que residen con sus familias.

Para la recogida de la información se ha diseñado un cuestionario que recoge, en primer lugar, información sobre la situación sociodemográfica de cada participante.

En segundo lugar se ha incluido el TMMS-24 (Fernández-Berrocal, Extremera y Ramos, 2004). Esta prueba está formada por 24 ítems, más bien breves, que cada persona tiene que valorar a través de una escala tipo Likert de cinco puntos. Esta escala recoge información sobre tres dimensiones fundamentales en la Inteligencia Emocional intrapersonal: Atención a los propios sentimientos, definida como el grado en que las personas prestan atención a sus sentimientos y emociones, Claridad emocional, se refiere a las formas cómo las personas perciben sus emociones y reparación de las propias emociones, referida a la capacidad para interrumpir los estados emocionales negativos y prolongar en el tiempo los positivos, siendo la escala de evaluación de Inteligencia Emocional más utilizada en los estudios desarrollados con población de habla hispana. En esta escala los 8 primeros ítems miden el factor Atención, los 8 siguientes el factor Claridad Emocional y los 8 últimos el factor Regulación Emocional.

En tercer lugar se ha incluido el RESI-m (Palomar \& Gómez, 2010) para recoger la información relativa a Resiliencia. Esta prueba consta de 43 ítems, con cinco opciones de respuesta de tipo Likert y evalúa 5 dimensiones: Competencia Personal, definida como el sentimiento de adecuación, eficiencia y competencia para afrontar retos y amenazas; Competencia Social, definida como la capacidad o habilidad para interactuar con éxito con el ambiente y lograr la satisfacción de necesidades; Coherencia Familiar, definida como el tiempo que comparte cada persona con su unidad familiar y la lealtad y fortaleza de esta relación; Apoyo Social, referida a los vínculos que cada 
persona tiene con las personas con las que comparte contexto; y Estructura Personal, definida como las reglas y las actividades que implementa cada persona para organizar y ordenar su vida.

La fiabilidad (consistencia interna establecida mediante el coeficiente alfa de Cronbach) de las pruebas utilizadas es alta y adecuada (0.83).
Todos los análisis estadísticos se han realizado con el paquete estadístico informático SPSS 20. Se ha utilizado la prueba t de Student para muestras independientes para contrastar las medias de los grupos de estudio, considerándose el nivel de significación estadística para $p \cdot .05$.

\section{Resultados}

Tabla 1: Comparación de medias en Inteligencia Emocional

\begin{tabular}{|l|c|c|c|c|c|}
\hline & $\mathrm{N}$ & $\begin{array}{c}\text { Inteligencia } \\
\text { Emocional }\end{array}$ & $\begin{array}{c}\text { Atención } \\
\text { emocional }\end{array}$ & $\begin{array}{c}\text { Claridad } \\
\text { emocional }\end{array}$ & $\begin{array}{c}\text { Regulación } \\
\text { emocional }\end{array}$ \\
\hline Residencia en Recursos de protección & 138 & 62.20 & 21.95 & 20.56 & 20.08 \\
\hline Residencia familiar & 270 & 85.84 & 27.68 & 27.42 & 30.73 \\
\hline Diferencia de medias & & 23.23 & 5.73 & 6.85 & 10.64 \\
\hline Sig. Bilateral & & .000 & .000 & .000 & .000 \\
\hline
\end{tabular}

Los resultados que presenta la Tabla 1 muestran diferencias estadísticamente significativas en las puntuaciones medias en Inteligencia Emocional y en las tres habilidades que conforman el cuestionario empleado: Atención, Claridad y Regulación Emocional, entre los grupos de personas tutelados por la administración y que residen en un recursos de protección y las personas que viven en su residencia familiar y que, además, no tienen ninguna medida ni proceso de intervención en servicios sociales, donde el segundo grupo obtiene unas puntuaciones significativamente más altas en todas las variables de estudio.

Estos datos muestran que las mayores diferencias se producen en la variable Regulación emocional, siendo, además, la Regulación emocional la variable con mayor puntuación media en el grupo de personas que viven en su residencia familiar y la variable con menor puntuación media entre las personas que residen en recursos de protección y están tuteladas por la administración.

\begin{tabular}{|l|c|c|c|c|c|c|c|}
\hline \multicolumn{7}{|c|}{ Tabla 2: Comparación de medias en Resiliencia } \\
\hline & $\mathrm{N}$ & Resiliencia & Fortaleza & Competencia & $\begin{array}{c}\text { Apoyo } \\
\text { Familiar }\end{array}$ & $\begin{array}{c}\text { Apoyo } \\
\text { Social }\end{array}$ & Estructura \\
\hline $\begin{array}{l}\text { Residencia en Recursos } \\
\text { de protección }\end{array}$ & 138 & 133.47 & 55.80 & 24.08 & 18.52 & 14.63 & 15.47 \\
\hline Residencia familiar & 270 & 139.00 & $58-08$ & 25.22 & 19.73 & 16.24 & 14.33 \\
\hline Diferencia de medias & & 5.52 & 2.27 & 1.13 & 1.21 & 1.60 & 1.14 \\
\hline Sig. Bilateral & & .029 & .062 & .090 & .027 & .009 & .005 \\
\hline
\end{tabular}

Los resultados presentados en la tabla 2 muestran que, el grupo de personas que reside en un recursos de protección obtiene puntuaciones significativamente inferiores al grupo que reside con sus familias y no tiene contacto con los servicios sociales. En cuanto a cada una de las dimensiones del cuestionario, el grupo de personas que residen con sus familias obtienen puntuaciones más altas en Resiliencia en todas las dimensiones excepto en Estructura, aunque estas diferencias no son significativas en todas estas dimensiones. Encontramos diferencias significativas con puntuaciones 
más altas para el grupo de personas que residen con sus familias en Resiliencia, Apoyo Social y Apoyo Familiar, y a favor del grupo de personas que residen en un centro de protección en la dimensión Estructura. No encontramos diferencias significativas en las puntuaciones encontradas en las dimensiones de Fortaleza y Competencia.

Los resultados encontrados nos permiten confirmar nuestras hipótesis de trabajo planteadas en el caso de la Inteligencia Emocional. En el caso de la Resiliencia nuestra hipótesis se confirma, puesto que las diferencias son significativas en Resiliencia, pero con los matices ya comentados para cada una de las dimensiones que conforman el cuestionario empleado para medir Resiliencia.

\section{Discusión y conclusiones}

Del análisis de nuestros datos, y en relación a los resultados encontrados en Inteligencia Emocional, se desprenden diferentes conclusiones. En primer lugar, los resultados presentan la necesidad de trabajar en el desarrollo de las habilidades emocionales de las personas que residen en un recurso de protección. Desde nuestra perspectiva, sistémica y ecológica, consideramos que obtendremos mejores resultados si abordamos esta acción desde una doble dimensión: trabajando directamente con los menores que residen en estos recursos, desarrollando y fomentando sus habilidades emocionales y trabajando, también, con los trabajadores, educadores y responsables de la educación y cuidados de estos menores, puesto que en la mayoría de los casos estos trabajadores son las principales figuras de apego y referentes, también emocionales, de los menores bajo su responsabilidad.

En segundo lugar, si bien es verdad que las puntuaciones son significativamente inferiores en el caso de las personas que residen en recursos de protección en las tres habilidades emocionales que incluye nuestra prueba (Atención, Claridad y Regulación emocional), estas diferencias son más pronunciadas y significativas en el caso de la Regulación emocional. Según estos datos, la especial dificultad para regular las emociones, y por tanto para adecuar el comportamiento a la situación en la que el menor se encuentra, podría explicar, al menos en parte, y estar en la base, junto con otras variables, los motivos por los que los menores que pasan por un recurso de protección pasan también, o tienen más probabilidades de pasar, por algún recurso de reforma. En todo caso, sea cierta o no esta percepción, parece evidente que el grupo de menores residentes en recursos de protección presentará mayores dificultades con la regulación de sus emociones y por tanto con la elección del comportamiento más adecuado a cada situación.

En tercer lugar, y aunque el grupo control obtenga puntuaciones significativamente más altas que el grupo experimental, parece, también, evidente la necesidad de incluir la emoción y contenidos emocionales en los institutos y colegios. Por varios motivos: A institutos y colegios también asisten personas con especiales dificultades en inteligencia emocional y que no tienen una familia estable (en el tiempo ni en las personas que la componen), con un entorno cambiante en cuanto a referentes y figuras de apego, que no siempre tienen la oportunidad de contar con una figura adecuada para el desarrollo emocional. Además, que las puntuaciones sean más altas en los menores que residen con sus familias, no significa que éstas sean altas con respeto a los indicadores del cuestionario o no pudieran ser mejores, y por lo tanto, aumentar las probabilidades de éxito de toda la población. Por último, es hora de normalizar e incluir los contenidos emocionales en los planes de estudio, como forma, también, de visibilizar y dotar de la importancia que merecen estos contenidos. Consideramos, que si el objetivo del sistema educativo es, precisamente, educar, dejar fuera del sistema el mundo emocional es olvidarse de parte fundamental del desarrollo de la persona.

Por tanto, es muy probable que los menores residentes en recursos de protección encuentren, atendiendo a las puntuaciones significativamente inferiores en Inteligencia Emocional y sus tres categorías, mayores dificultades para alcanzar el éxito académico (Ferragut \& Fierro, 2012; Jiménez Morales \& López Zafra, 2009), tengas mayores dificultades para encontrar un empleo (Solano-Gómez, 2013) o presenten un bienestar psicológico inferior (Salguero, Palomera \& Fernández-Berrocal, 2012).

En cuanto a los resultados obtenidos para la variable Resiliencia encontramos, de nuevo, que la población de menores residentes en recursos de protección obtiene puntuaciones significativamente más bajas que la población de menores que no tienen relación alguna con los servicios sociales, aunque con matices. Estas puntuaciones presentan al grupo de menores tutelados por la administración y residente en un recurso de protección como un grupo de personas que tiene, por norma, que enfrentar y afrontar situaciones de mayor adversidad con menos herramientas de gestión emocional y Resiliencia. Esta afirmación presenta algunos matices. Hemos encontrado que los menores residentes en centros de protección presentan una mejor puntuación, estadísticamente significativa además, en la dimensión Estructura, 
que hacía referencia a las normas y formas de organizar la vida, lo que indica, o podría indicar, que los centros de protección son capaces de facilitar y proporcionar normas y la organización de las vidas durante, al menos, el periodo que pasan en el recurso. Nos preguntamos en todo caso si esta información acaba de ser positiva o no para estos menores, que al llegar a los 18 ven como desaparece esta estructura y organización de la que formaban parte para tener que crear y construir la suya propia sin, según los resultados de este estudio, todas las herramientas necesarias o, cuanto menos, con menos herramientas que los menores que residen con sus familias y no tienen contacto con los servicios sociales.

Sin embargo, dadas las puntuaciones en Apoyo Social y Apoyo Familiar, parece que los centros no son capaces de dotar a las personas que residen en este tipo de recursos de relaciones sociales y familiares adecuadas o que, al menos, fomenten su Resiliencia, y en todo caso, no en la medida en que las familias externas y ajenas a los servicios sociales lo proporcionan. No podemos perder de vista el hecho de que la función básica del recurso de protección es sustituir a la familia de origen y crear y facilitar nuevas relaciones de apego y figuras de referencia para los menores que allí residen. Según estos resultados, esto no se está consiguiendo, o no en la medida en la que las familias sin contacto con los servicios sociales lo hacen son sus menores, dejando, por tanto, a los menores que provienen de recursos de protección en una situación de desventaja ante una situación, insistimos, de mayor adversidad.

Por otro lado, es justo el apoyo social lo que podría perdurar en el tiempo al finalizar la estancia en el recurso de protección, y según nuestros resultados este apoyo se esta generando el una medida significativamente inferior a la que presentan los menores que residen con sus familias sin contacto con los servicios sociales. En cuanto a las relaciones sociales, aún hoy, los menores residentes en centros de protección mantienen el estigma de pertenecer a un recurso social, de protección en este caso, lo que condiciona, como mínimo al principio, la forma en la que la población se relaciona con estas personas. Esto puede significar que, en lo relativo a la dimensión de Apoyo Familiar, toda, o casi toda, la responsabilidad recae en el propio recurso y sus trabajadores y gestores, aunque también en la administración que los evalúa. En el caso del apoyo social, en cambio, la responsabilidad es compartida entre centro de protección, profesionales que allí trabajan y administración, pero también con el resto de instituciones y servicios por el que transitan estas personas: escuelas, institutos, centros médicos y recursos de la localidad, por lo que las posibles acciones a implementar tendrían que tener en consideración a todos los agentes que participan de este apoyo social y en los procesos educativos de estos menores tutelados por la administración y residentes en recursos de protección.

Ignorar estos resultados, si se confirman y mantienen en el estudio de poblaciones más amplias y representativas y considerando los resultados que presentan diferentes estudios sobre Resiliencia y sus efectos en diversos ámbitos de la persona y su entorno, puede suponer que estamos limitando, de nuevo, las posibilidades de éxito académico de los menores que residen en recursos de protección (Gaxiola, González, Contreras y Gaxiola, 2012) disminuyendo las posibilidades de éxito y superación de la situación especialmente adversa de estos menores (Rutter, 2007) y perpetuando su situación de exclusión, negándoles algunas de las herramientas para su desarrollo (Masten, 2007).

Dada la propia definición de Resiliencia y su carácter multidimensional (Palomar y Gómez, 2010) así como la diversidad de variables personales y ambientales de las que depende, se nos presentan y facilitan diversos ámbitos donde la intervención para aumentar las puntuaciones en Resiliencia de estos menores que residen en recursos de protección es posible: formación de los trabajadores y gestores de los recursos de protección en relación al Apoyo Familiar; También coordinación y sensibilización de los recursos de lo que forman parte las personas que residen en un centro de protección; detectar y evaluar en cada caso, en cada menor concreto cuáles de sus fortalezas y cuales sus debilidades, para desarrollar las primeras y mejorar las segundas. Se trata de un trabajo integral, de nuevo ecológico y sistémico (en cuanto que incluye a la persona y su entorno) y no sólo de la persona en la que queremos desarrollar su Resiliencia.

\subsection{Dificultades del diseño.}

Este diseño presenta algunas limitaciones relativas, primero, a la no existencia, o negación del permiso para consultarlas, de estadísticas relativas al número de menores de protección que ahora están en reforma. En segundo lugar, nos encontramos con dificultad para tener acceso a los centro de protección por cuestiones legales, de regulación interna de los recursos, diferentes condiciones para acceder a los diferentes recursos según localización y responsables políticos y técnicos del mismo. En este sentido el tamaño de la muestra ha estado condicionado con las imposibilidad de acceder a todos los centros de 
protección y en todas las administraciones con las que se ha contactado. Por último, en relación con la situación de parte de la muestra, aparecen limitaciones relacionadas con la manera de no sesgar la información obtenida en el grupo control y que no tuvieran contacto con los servicios sociales, porque no podemos determinar si lo tendrán en el futuro.

En cuanto al tamaño de la muestra, es representativa de los lugares seleccionados pero no del conjunto de recursos de protección de España, por lo que nos sirve para tener acceso a una primera evaluación diagnóstica acerca de cómo puede estar la situación, y al confirmar nuestra hipótesis darnos una dirección y sentido en la que seguir investigando con el objeto de, al final, mejorar las condiciones con las que los menores tutelados se enfrentan a su futuro al cesar las medidas con su mayoría de edad. Por tanto, estos resultados, a priori, no son representativos de la población de menores que residen en algún recurso de protección en todo el país.

Por último el oscurantismo con que los responsables de la sección de protección y reforman tratan los datos relativos, por ejemplo, a las medidas previas de las personas que están en reforma, pero también, las dificultades presentadas para evaluar y valorar la situación de los menores que residen en protección, ha sido una dificultad importante en el diseño de este trabajo, y que, creemos, presenta una solución muy sencilla.

\subsection{Posibles línea de trabajo}

Consideramos necesario y muy relevante para el futuro de las personas menores de edad que están tutelados por la administración y residen en recursos de protección avanzar en el estudio de su situación en cuánto a las habilidades con las que cuentan para enfrentarse con éxito a su emancipación del recurso e incluir no sólo a un mayor número de personas, con el objetivo de poder generalizar los resultados a toda la población, sino también incluir el estudio de otras variables que puedan explicar la situación con mayor precisión y, también, darnos información sobre posibles contenidos de trabajo en estos recursos y así aumentar las probabilidades de éxito de estas personas.

Nos planteamos también la necesidad de estudiar las variables que hacen que los jóvenes que pasan a reforma estén allí, procedan de protección o no, puesto que esta información nos podría ayudar a prevenir estas situaciones a través del trabajo de las mismas en los recursos correspondientes: centros de protección, familias de acogida, colegios, institutos y otras instituciones. Además, está información podría estar en la justificación del diseño de planes de trabajo, planes de estudio, y porqué no, legislación relativa a cuestiones educativas, menores y recursos que la administración implementa para hacer frente a las diversas situaciones que se presentan hasta la mayoría de edad de estos menores.

Por último, consideramos importante conocer la situación, las habilidades y capacidades que tienen las personas que viven con alguna otra medida de protección: en familias de acogida, en acogimiento familiar, con situación de riesgo declarada o algún otro proceso de intervención desde servicios sociales, para conocer cómo están en relación, en nuestro caso, a las habilidades emocionales y de Resiliencia, y si el trabajo que se realiza desde las familias de acogida o los propios recursos sociales resulta positivo, o no tan positivo, para las personas objeto de la acogida y la intervención.

\section{Referencias bibliográficas}

Bonanno, G. A. (2004) Loss, trauma and human resilience: Have we underestimated the human capacity to thrive after extremely aversive events? American Psychologist. 59(1), 20-28.

Carretero Bermejo, R. (2010). Resiliencia. Una visión positiva para la prevención e intervención desde los Servicios Sociales. Nómadas. Critical Journal of Social and Juridical Sciences. 27 (3).

Cyrulnyk, B. (2001). Los patitos feos. La resiliencia: una infancia infeliz no determina la vida. Madrid. Gedisa.

Fernández-Berrocal, P., Extremera, N. \& Ramos, N. (2004). Validity and reliability of the Spanish modified version of the Trait Meta-Mood Scale. Psychological Reports, 94, 751-755.

Ferragut, M., \& Fierro, A. (2012). Inteligencia emocional, bienestar personal y rendimiento académico en preadolescentes. Revista latinoamericana de Psicología, 44(3), 95-104.

Gartzia, L., Aritzeta, A., \& Balluerka, N. (2012). Inteligencia emocional y Género : más allá de las diferencias sexuales Introducción. Anales de Psicología, 28, 567-575.

Gaxiola, R., Frías, A, Hurtado A., Salcido, N., \& Figueroa, F. (2011). Validación del inventario de resiliencia (IRES) en una población del noroeste de México. Enseñanza e Investigación en Psicología, 16 (1), 73-83. 
Gaxiola, J., González, S., Contreras, Z., \& Gaxiola, E. (2012). Predictores del rendimiento académico en adolescentes con disposiciones resilientes y no resilientes. Revista de Psicología, 30 (1), 49-74.

Gómez, E., \& Kotliarenco, M. (2010). Resiliencia Familiar: un enfoque de investigación e intervención con familias multi-problemáticas. Revista de Psicología (Santiago), 19, 2-10.

Gorostiaga, A., Balluerka, N., \& Soroa, G. (2014). Evaluación de la empatía en el ámbito educativo y su relación con la inteligencia emocional. Revista de Educación, 364, 43- 61.

Jiménez Morales, M.I., \& López-Zafra, E. (2009). Inteligencia Emocional y rendimiento escolar: Estado actual de la cuestión. Revista latinoamericana de psicología, 2 (1), 69-79.

Lampel, J., Bhalla, A., \& Jha, P. P. (2014). Does governance confer organisational resilience? Evidence from UK employee owned businesses. European Management Journal, 32 (1), 66-72.

López Peláez, A. (2012). Profesión, ciencia y ciudadanía: retos para el Trabajo Social y los Servicios Sociales en el siglo XXI. Azarbe. Revista Internacional de Trabajo Social y Bienestar, 1, 61-71

López, M., Rosales, J., Chávez, M., Byrne, S., \& Cruz, J. (2009). Factores que influyen en el pronóstico de recuperación de las familias en riesgo psicosocial: el papel de la Resiliencia del menor. Psicothema, 21 (1), 90-96.

Manciaux, M. (2003). La resiliencia: resistir y rehacerse. Barcelona: Gedisa

Masten, A. S., (2007) Resilience in developing systems: Progress and promise as the fourth wave rises. Development and Psychopathology 19.921-930.

Masten, A. S. (2004) Regulatory Processes, Risk, and Resilience in Adolescent Development. Annals of the New York Academy of Sciences, 1021, 321-319.

Masten, A., \& Obradovic, J. (2006) Competence and resilience in development. Annals of the New York Academy of Sciences, 1094, 13-27

Mayer, J. D., \& Salovey, P. (1997). What is emotional intelligence? Emotional development and emotional intelligence: Implications for educators. New York: Basic Books.

Mayer, J. D., Salovey, P., \& Caruso, D. (2004). Emotional intelligence: Theory, findings and implications. Psychological Inquiry, 15, 197-215.

Muñoz-Silva, A. (2012). El estudio de la resiliencia desde la perspectiva evolutiva y su aportación a la comprensión del riesgo y la protección en la intervención social. Portularia. Revista de Trabajo Social, 12 (1), 9-16.

Palma-García, M., \& Hombrados-Mendieta, I. (2013). Trabajo Social y resiliencia: revisión de elementos convergentes. Portularia. Revista de Trabajo Social. 13 (2), 89-98

Palomar, J., \& Gómez, N.E. (2010). Desarrollo de una escala de medición de la resiliencia con mexicanos (RESI-M). Interdisciplinaria, 27, 7-22.

Rutter, M. (2007). Resilience, competence, and coping. Child Abuse \& Neglect, 3, 205-209.

Salguero, J.M., Palomera, R., \& Fernández-Berrocal, P. (2012) Perceived emotional intelligence as predictor of psychological adjustment in adolescents: a 1-year prospective study. European Journal of Psychology of Education, 27(1), 21-34.

Salovey, P., \& Grewal, D. (2006). Inteligencia Emocional. Mente y Cerebro, 16, 10-20.

Salovey, P., \& Mayer, J. D. (1990) Emotional intelligence. Imagination, Cognition, and Personality, 9 (3), 185-211.

Salovey, P., Mayer, J. D., \& Caruso, D. (2002). The positive psychology of emotional intelligence. In C. R. Snyder, y S. J. Lopez (Eds.). The Handbook of positive psychology. New York: Oxford University Press.

Solano-Gómez, A. (2013). Inteligencia Emocional en el trabajo. Sus implicaciones y el rol de la psicología laboral. Humanitas. 10 (10). 201-214.

Vera, B., Carbelo, B., \& Vecina, M.L. (2007) La experiencia traumática desde la Psicología Positiva: Resiliencia y crecimiento postraumático. Papeles del psicólogo, 27. 40-49. 


\section{CÓMO CITAR ESTE ARTÍCULO}

Carretero-Bermejo, R. (2018). Resiliencia e inteligencia emocional. Variables de protección en menores residentes en recursos de protección. Pedagogía Social. Revista Interuniversitaria, 32 139-149. DOI:10.7179/PSRI_2018.32.10

\section{DIRECCIÓN COMPLETA DE LOS AUTORES}

Raúl Carretero Bermejo. Plaza de Agustín Salido, 7, 10 A 13003 Ciudad Real.

info@raulcarreterobermejo.com

\section{PERFIL ACADÉMICO}

Raúl Carretero Bermejo. Doctor en Educación por la UNED con la tesis titulada "Inteligencia Emocional y Sexismo. Aproximación al componente emocional del Sexismo" en la que obtiene la calificación de Sobresaliente con mención Cum Laude. Licenciado en Psicopedagogía en la UCLM y diplomado en Magisterio. Ha trabajado como profesor asociado en la Facultad de Ciencias de la Educación de Cuenca, UCLM, en el grado de Educación Social. 
